TOKYO J. MATH.

VoL. 18, No. 1, 1995

\title{
The Involutions of Compact Symmetric Spaces III
}

\author{
Tadashi NAGANO and Makiko Sumi TANAKA
}

Sophia University and International Christian University

\section{Introduction.}

In this part, we will construct certain fibrations intended for study of differential geometry, especially that of Riemannian submersions. Their base manifolds are polars and the fibres are centrioles of the meridians (See Section 4 for the review of these terms). They are listed in Table II, which include such significant examples as various twistor spaces of Calabi, Penrose and others as well as the Hopf fiberings; we will briefly explain more recent twistor spaces of $\mathbf{R}$. Bryant [Br] in Section 8. As an application we will study and classify the simple graded algebras

$$
\mathfrak{g}=\mathfrak{g}_{-2}+\mathfrak{g}_{-1}+\mathfrak{g}_{0}+\mathfrak{g}_{1}+\mathfrak{g}_{2}
$$

of this type (7.1). That was done by S. Kaneyuki ([K], [KA]) already, but we will translate the problem into that of the compact form of $g$ (Prop. 7.6) and then describe it geometrically in terms of two tiers of fibrations (7.10); the relevant manifolds such as the fibre of one fibration are the fixed point sets of an involution acting on those of the other. The translation is so satisfactory it gives some insight into the gradation and the classification becomes a matter of picking up certain 2-tiered fibrations as Theorem 7.10 indicates (See 7.14 for the comparison with Kaneyuki's table, which agrees with our results). We return to the noncompact $g$ and interpret its gradation in terms of an affine symmetric space (7.12 \& 13). We will establish basic facts $(5.9,5.11$, etc.) in Section 5. We begin this part of the series with Section 4 in continuation of [N67] for the convenience of quoting items in it.

We thank Prof. Soji Kaneyuki warmly for valuable informations.

\section{Definitions, notations and conventions.}

4.1. We denote by $F(\tau, M)$ the fixed point set of a transformation $\tau$ of $M$; similarly, for a set $S$ of transformations, $F(S, M)$ denotes the common fixed points of the members of $S$. A symmetric space, or just a space, is a manifold $M$ with the (point) symmetry $s_{x}$ 
at every point $x$ such that (1) the singleton $\{x\}$ is open in $F\left(s_{x}, M\right),(2) s_{x}$ is involutive and (3) $M$ admits an affine connection $\nabla$ which every symmetry $s_{x}$ leaves invariant.

4.2. A homomorphism (of a symmetric space) $f: M \rightarrow N$ is a smooth map of a symmetric space $M$ into another which commutes with the symmetries; one has $s_{f(x)} \circ f=f \circ s_{x}$ for every point $x$ of $M$. Thus a geodesic is a homomorphism $c: R \rightarrow M$ of the real line. We have a category of the symmetric spaces and the homomorphisms between them. A subspace $M \subset N$ is a symmetric space of which the inclusion is a homomorphism. The automorphism group of $M$ is denoted by $I(M)$ and its connected component $I(M)_{(1)}$ through the unit element 1 is denoted by $G$, or $G_{M}$ or $G(M)$; generally, we denote by $X_{(p)}$ the connected component of $X$ through the point $p$. We may follow the practice of using $G$ to denote its covering group when it is convenient, especially in describing specific spaces such as the complex Grassmann manifold $G_{r}\left(C^{n}\right)=\mathrm{SU}(n) /$ $\mathrm{S}(\mathrm{U}(r) \times \mathrm{U}(n-r))$; i.e. $G$ may not be effective on $M$. If $M$ is connected as we usually assume, $G$ is transitive on $M ; M=G / K, K$ the stabilizer at a point $o$, and $K$ is open in $F\left(\operatorname{ad}\left(s_{o}\right), G\right)$. When $M$ happens to be a Lie group, we call a gr-homomorphism a homomorphism of $M$ as a Lie group. Every local isomorphism class of connected simple spaces $M$ contains a unique space $M \%$ of which every $M$ in the class is a covering space. We call $M^{\%}$ the bottom space (the adjoint space in $\left.[\mathrm{H}]\right) ; M^{\%}=G \% / F\left(\operatorname{ad}\left(s_{o}\right), G \%\right)$. We write $M^{\sim}$ for the universal covering space of $M$. We say $M$ is locally isomorphic with $N$ if $M^{\sim}$ is isomorphic with $N^{\sim}$; the symbol is $M \fallingdotseq N$.

4.3. We assume compactness of $M$, unless otherwise mentioned. Thus the uniqueness of $\nabla$ implies that $\nabla$ is the Levi-Civita connection of a Riemannian metric which $I(M)$ leaves invariant (provided $M$ is simple). Another consequence is that $M$ is a local product of a torus and simple spaces, if $M$ is connected.

We will freely use known facts in Bourbaki [B] and Helgason [H].

We now recall and explain definitions of basic terms.

4.4. We call every component of $F\left(s_{o}, M\right) a$ polar of $o$ in $M$ and denote it by $M^{+}$ or $M^{+}(p)$ if it contains a point $p$. We also call it a pole when it is a singleton $M^{+}(p)=\{p\}$.

4.4a. Every homomorphism carries a polar into another.

4.4b. If $M$ has a pole $p$ of $o$, then $M$ admits a double covering homomorphism onto another space $M /\{o, p\}$ which carries $\{o, p\}$ onto a single point.

4.5. Given a subspace $N$, we call a subspace $N^{\perp}$ the c-orthogonal space to $N$ at $p$ if the tangent space $T_{p} N^{\perp}$ is the orthogonal complement of $T_{p} N$ in $T_{p} M$. If a subspace $N$ equals $F(\tau, M)_{(o)}$ for some involution $\tau$, then $N^{\perp}:=F\left(\tau \circ s_{o}, M\right)_{(o)}$ is c-orthogonal to $N$ at $o$. One notes that the intersection $N \cap N^{\perp}$ is then contained in $F\left(s_{o}, M\right)$ and hence in $F\left(s_{o}, N\right)$; in case $F(\tau, M)$ is connected one has the equality $N \cap N^{\perp}=F\left(s_{o}, N^{\perp}\right)$.

4.6. The c-orthogonal space $M^{-}(p)$ to a polar $M^{+}(p)$ at $p$ is called the meridian 
to $M^{+}(p)$ at $p$. The $K_{(1)}$-congruence class of $M^{-}(p)$ is independent of $p$, depending only on $M^{+}(p)$.

4.6a. $\quad p$ is a pole of $o$ in $M^{-}(p)$.

4.6b. The rank $r\left(M^{-}(p)\right)$ equals $r(M)$ (See 1.8).

4.7. Given a pole $p_{o}$ of $o$ in $M$, the double covering morphism $\pi: M \rightarrow M^{\prime \prime}$ which carries $p_{o}$ into $\pi(o)$ (See 4.4b) projects the polars of $o$ onto those of $\pi(o)$ in $M^{\prime \prime}$ by (4.4a), but the inverse images of the polars of $\pi(o)$ are either polars or what we call the centrioles for the pair $\left(o, p_{o}\right)$. The centrioles may be characterized as the connected components of the set of the midpoints of all the geodesic segments joining $o$ to $p_{o}$, called the centrosome for $\left(o, p_{o}\right)$ and denoted $C\left(o, p_{o}\right) . C\left(o, p_{o}\right)$ is a subspace.

4.8. A member $p$ of a group $G$ is called pre-involutive iff $p$ lies in a centriole for the pair $\left(1, p_{o}\right)$ of 1 and a pole $p_{o}$; equivalently, $p^{2}$ is an involutive member of the center of $G$. While the polars of 1 in $G$ are the conjugate classes of the involution in $G$, the centrioles are those of the pre-involutions.

Basic importance of the polar and the meridian should be obvious from the fact that a connected space is completely determined by any single pair of a polar and the corresponding meridian (1.15).

Definitions of $\varepsilon, l_{k}, \delta_{r}, \eta_{r}$ and $\zeta_{r}$. We propose to fix standard notations for involutions in simple groups which are common to different groups and which we find are convenient especially in dealing with the exceptional groups.

4.9. First we denote by $\varepsilon$ the generator of the kernel of the projecion $\pi$ of the spin group $\mathrm{SO}(n)^{\sim}$ onto $\mathrm{SO}(n)=\mathrm{SO}(n)^{\sim} /\{1, \varepsilon\}, n>2$.

4.10. We next denote by $l_{k}, k$ odd, the pre-involutive members with the squares $=\varepsilon$ in $\mathrm{SO}(n)^{\sim}$, which lie in the centrioles $G_{2 k}\left(R^{n}\right)^{\sim}$ and project into $I_{2 k}:=-1_{2 k} \oplus 1_{n-2 k}$ (the diagonal with the eigenvalue -1 of multiplicity $2 k$ ) of $\mathrm{SO}(n)$; hence the projection $\pi\left(G_{2 k}\left(R^{n}\right)^{\sim}\right)$ is the Grassmann manifold $G_{2 k}\left(R^{n}\right)$ which is thus the polar $\left[l_{k}\right]$ containing $I_{2 k}$.

4.11. The center of $\mathrm{SO}(2 r)^{\sim}$ is generated by $\varepsilon$ and $\delta_{r}$ (which lies in the direction of the $r$ th fundamental weight $\varpi_{r}$; we adopt the numbering in Bourbaki [B] for weights and roots). In case $r$ is even, $\delta_{r}$ is involutive (but one has $\delta_{r}^{2}=\varepsilon$ for an odd $r$ ). We choose pre-involutive points $\eta_{r}$ and $\zeta_{r}$ in the directions of $\varpi_{r}$ and $\varpi_{r-1}$ respectively so that the centrioles for $\left(1, \delta_{r}\right)$ are $\left[\eta_{r}\right]=\left[\delta_{r} \eta_{r}\right]$ and $\left[\varepsilon \eta_{r}\right]$ both of which are isomorphic with $\operatorname{DIII}(r)$; and similarly for the centrioles $\left[\zeta_{r}\right]$ and $\left[\varepsilon \zeta_{r}\right]$ for $\left(1, \delta_{r} \varepsilon\right)$. One has $\pi\left(\delta_{r}\right)=I_{2 r}$ and the centrosome $\operatorname{OIII}(r)$ for $\left(1, I_{2 r}\right)$ in $\mathrm{SO}(2 r) ; \pi\left(\eta_{r}\right)$ was denoted by $J_{r}$ in (3.3).

4.12. Back to $\varepsilon$, this is independent of $n$ in that $\varepsilon$ is carried into $\varepsilon$ by the inclusion map: $\mathrm{SO}(n)^{\sim} \rightarrow \mathrm{SO}(n+1)^{\sim}$ induced by the natural inclusion map: $\boldsymbol{R}^{n} \rightarrow \boldsymbol{R}^{n} \oplus \boldsymbol{R}=\boldsymbol{R}^{n+1}$. More generally, we use local gr-monomorphisms of Dynkin index $=1$ (or, equivalently, those which induce isomorphisms on the third homotopy groups $\pi_{3}$, [AHS]) in order 
to define the polars $[\varepsilon],\left[\delta_{r}\right]$ and so forth in simple groups. One knows these gr-monomorphisms of Dynkin index 1 ([AHS] and [MT]):

4.13. $\mathrm{SO}(n)^{\sim} \rightarrow \mathrm{SO}(n+1)^{\sim}, \mathrm{SU}(n) \rightarrow \mathrm{SO}(2 n), \mathrm{Sp}(n) \rightarrow \mathrm{SU}(2 n)$, and

4.13a. $\mathrm{G}_{2} \rightarrow \mathrm{SO}(9)^{\sim} \rightarrow \mathrm{F}_{4} \rightarrow \mathrm{F}_{6} \rightarrow T \cdot \mathrm{E}_{6} \rightarrow \mathrm{E}_{7} \rightarrow \mathrm{Sp}(1) \cdot \mathrm{E}_{7} \rightarrow \mathrm{E}_{8}$, where $T:=\mathrm{U}(1)$ and - means a certain obvious local product (1.5b).

4.14. $\mathrm{SO}(n)^{\sim}, n \geqq 4$, has the polars $\left\{\left[\delta_{2 s}\right] \mid 4 \leqq 4 s \leqq n\right\}$ plus $\{[\varepsilon],[1]\}$ and, in case $n=2 r$ and $r$ are even, $\left[\delta_{r} \varepsilon\right]$ is added. For $\operatorname{SO}(n),\left\{\left[l_{k}\right] \mid k\right.$ odd and $\left.2 \leqq 2 k \leqq n\right\}$ is added with $[\varepsilon]$ deleted. While the inclusion: $\mathrm{U}(n) \rightarrow \mathrm{SO}(2 n)$ gives a bijection of the set of the polars, the one: $\mathrm{SU}(n) \rightarrow \mathrm{SO}(2 n)$ is factored through $\mathrm{SU}(n) \rightarrow \mathrm{SO}(2 n)^{\sim}$ and the projection, giving rise to a bijection onto $\left\{\left[\delta_{2 s}\right] \mid 4 \leqq 4 s \leqq n\right\}$ plus [1], which is similarly true for $\mathrm{Sp}(n) \rightarrow \mathrm{SU}(2 n)$. Thus the inclusions $\mathrm{Sp}(n) \rightarrow \mathrm{SU}(2 n) \rightarrow \mathrm{SO}(4 n)$ induce the ones $G_{s}\left(H^{n}\right) \rightarrow$ $G_{2 s}\left(C^{2 n}\right) \rightarrow G_{4 s}\left(R^{4 n}\right)$ of the polars denoted by the same symbol $\left[\delta_{2 s}\right]$.

4.15. The polars of 1 in the 1-connected exceptional groups are $\left\{[\varepsilon],\left[\delta_{2}\right]\right.$, $[1]\} \subset \mathbf{E}_{6},\left\{[\varepsilon],\left[\delta_{2}\right],\left[\delta_{6}\right],[1]\right\} \subset \mathbf{E}_{7},\left\{[\varepsilon],\left[\delta_{2}\right],[1]\right\} \subset \mathbf{E}_{8},\left\{[\varepsilon],\left[\delta_{2}\right], \cdot[1]\right\} \subset \mathbf{F}_{4}$, and $\left\{\left[\delta_{2}\right],[1]\right\} \subset \mathrm{G}_{2}$; more concretely, $[\varepsilon]$ is EIII in $\mathrm{E}_{6}, \mathrm{EVI}$ in $\mathrm{E}_{7}$, EVIII in $\mathrm{E}_{8}$, while $\left[\delta_{2}\right]$ is EII in $E_{6}$, EVI (which is distinct from the other one) in $E_{7}$, and EIX in $E_{8}$ respectively, $\left[\delta_{6}\right]$ being a pole in $\mathrm{E}_{7}$; in order to denote the pole of $\mathrm{E}_{7}$ by $\left[\delta_{6}\right]$, we have chosen the inclusions $\mathrm{E}_{7} \leftarrow \mathrm{Sp}(1) \cdot \mathrm{SO}(12)^{\sim} \rightarrow \mathrm{SO}(4)^{\sim} \cdot \mathrm{SO}(12)^{\sim} \rightarrow \mathrm{SO}(16) \#:=\mathrm{SO}(16)^{\sim} /$ $\left\{1, \delta_{8} \varepsilon\right\} \rightarrow \mathrm{E}_{8}$ as well. One has to specify the inclusions to extend the definitions of $\left[\delta_{r}\right]$ to other groups, because outer involutive automorphisms (which have the Dynkin index $=1$ ) of $\mathrm{SO}(2 r)^{\sim}$ exchange $\delta_{r}$ and $\delta_{r} \varepsilon$.

4.16. Notations of symmetric spaces. We have been using standard symbols with slight modifications such as $T=\mathrm{U}(1)$ as in $\mathrm{U}(n)=T \cdot \mathrm{SU}(n), \mathrm{UI}(n)=\mathrm{U}(n) / \mathrm{O}(n)=T \cdot \mathrm{AI}(n)$ and $\mathrm{UII}(n)=\mathrm{U}(2 n) / \mathrm{Sp}(n)=T \cdot \operatorname{AII}(n)$. $\mathrm{OIII}(n)$ denotes $\mathrm{O}(2 n) / \mathrm{U}(n)$ which is the union of 2 copies of $\operatorname{DIII}(n)=\operatorname{SO}(2 n) / \mathrm{U}(n)$.

\section{Theorems.}

The polars in $M$ are deeply related to the topology of $M$; the next theorem is a first evidence (stated in an earlier paper without proof).

5.1 THEOREM. A compact connected symmetric space is orientable if and only if all the polars have even dimensions.

ProOF. Let $M$ be an orientable space. For every polar $M^{+}(p)$ of $o$, the quadratic transformation $Q_{o}(p):=s_{p} \circ s_{o}$ is homotopic with the identity $1_{M}$ on one hand and acts on the tangent spaces $T_{p} M^{-}(p)$ and $T_{p} M^{+}(p)$ as the identity and the negative identity respectively on the other hand. Therefore the dimension of $M^{+}(p)$ must be even since it equals the multiplicity of the eigenvalue -1 of $Q_{o}(p)$ acting on $T_{p} M$. Now assume 
that $M$ is not orientable. Then there is a double covering space $M^{\wedge}$ which is orientable; we denote by $\pi$ the projection: $M^{\wedge} \rightarrow M$. Let $\gamma: M^{\wedge} \rightarrow M^{\wedge}$ be the corresponding covering transformation; $\gamma$ reverses the orientation and carries every point $o^{\wedge}$ into its pole $\gamma\left(o^{\wedge}\right)$. Every centriole $C^{\wedge}$ for the pair $\left(o^{\wedge}, \gamma\left(o^{\wedge}\right)\right)$ projects onto a polar $C$ of $o=\pi\left(o^{\wedge}\right)$ in $M$. Now we choose $C^{\wedge}$ so that $C^{\wedge}$ meets one of the shortest circles, $c^{\wedge}$, passing through $o^{\wedge}$ and $\gamma\left(o^{\wedge}\right)$. Let $p^{\wedge}$ be the midpoint of the subarc of $c^{\wedge}$ between $o^{\wedge}$ and $\gamma\left(o^{\wedge}\right)$; $C^{\wedge}$ then meets $c^{\wedge}$ at $p^{\wedge}$. And $Q_{o^{\wedge}}\left(p^{\wedge}\right)$ stabilizes $c^{\wedge}$, carrying every point $\in c^{\wedge}$ into its pole in $c^{\wedge}$. Thus $\gamma \circ Q_{0^{\wedge}}\left(p^{\wedge}\right)$ fixes $c^{\wedge}$ pointwise and reverses the orientation of $M^{\wedge}$; in particular it reverses the orientation of $T_{p^{\wedge}}\left(M^{\wedge}\right) . \gamma \circ Q_{o^{\wedge}}\left(p^{\wedge}\right)$ can act on $M$ as $Q_{o}(p), p=\pi\left(p^{\wedge}\right)$. Since $Q_{o}(p)$ reverses the orientation of $T_{p} M$, it does that of $T_{p} C$ also. Therefore $C$ has an odd dimension by the above arguments.

5.2 COROLlaRY. Every polar in a connected group is even dimensional.

5.3. For another example, we point out that the cohomology group of a compact Kähler symmetric space can be easily read off from those of the polars (and the dimension of a certain factor space of each meridian) ([CS], [Ts]).

A meridian in $M$ has the curvature or the root system closely related to that of $M$ as is explained in 5.5; hence even their noncompact forms can not only be defined but play an important part (Cf. Mok and Tsai, or Tsai [T]).

5.4 THEOREM. The following conditions (i) through (iv) are equivalent to each other for a compact, connected space $M=G / K$ :

(i) $M$ is isomorphic with a polar of 1 or a centriole for $(1, x)$ in a covering group $G^{\wedge}$ of $G$,

(ii) The involution $\sigma:=\operatorname{ad}\left(s_{o}\right)$ is inner $(=\operatorname{ad}(b)$ for some involutive or pre-involutive member $b$ of $G$ ),

(iii) The Euler number $\chi(M)>0$, and

(iv) The rank $r(K)$ equals $r(G)$.

Proof. The equivalences between (ii), (iii) and (iv) are well known. So assume (i): $M$ is a polar or a centriole in a group $G^{\wedge}$. Let $p$ be a point of $M$. Then $M$ is the orbit $\operatorname{ad}\left(G^{\wedge}\right)(p)$ of the isotropy subgroup at 1 . If $M$ is a polar, the meridian $G^{\wedge}-(p)$ is $F\left(Q_{o}(p), G^{\wedge}\right)_{(1)}, Q_{o}(p)$ defined in the proof of 5.1 , and one has $M=G^{\wedge} / K$, where $K$ contains $G^{\wedge}-(p)$ as $K_{(1)}$. Thus (ii) follows, since $Q_{o}(p)$ is homotopic with 1 . If $M$ is a centriole, $M$ projects onto a polar in the group of which $G^{\wedge}$ is a double covering, (See 4.7), and (ii) obtains similarly. Assume (ii) for the converse. If $b$ is involutive [resp. pre-involutive], the orbit $\operatorname{ad}(G)(b)$ is a polar of 1 [resp. a centriole for $\left(1, b^{2}\right)$ ] and locally isomorphic with $G / F(\operatorname{ad}(b), G)=G / F\left(\operatorname{ad}\left(s_{o}\right), G\right)$ and hence with $M$. To reach the global conclusion, one has only to recall the fact 1.9: $M=G / K$ is a subspace of some covering group $G^{\wedge}$ of $G$ (if $G$ is effective).

5.4a Remark. One cannot drop the centriole from (i), since DIII( $r$ ) can show up 
only as a centriole in a group $\fallingdotseq S O(2 r)$ if $r$ is even.

5.5. We defined the roots as the nonzero eigenvalues of the Jacobi operators for the geodesics (2.3); they are the same as the usual roots or the restricted ones, except that we do (or should) not usually distinguish a root $\alpha$ from $-\alpha,(2.2)$. Thus the next lemma is almost immediate. For its setting, consider a geodesic $c$ in $M=G / K$; $c(t)=\exp (t \pi H)(o)$, where $o=c(0)$ and $H$ is a transvection $\left(\operatorname{ad}\left(s_{o}\right) H=-H\right.$, that is $)$ in the Lie algebra $\mathscr{L} G$ which lies in a Weyl chamber. The members of $\mathfrak{f}$, thought of as vector fields on $M$, furnish the solutions of the Jacobi equation for $c$ if restricted on $c$ ("variational completeness").

5.6 LEMMA. Let $c$ be a geodesic in a simple space $M$ and passing through o and $p:=c(1)$ as in the above. Then

(i ) $p($ or $t=1)$ is a conjugate point of o on $c \Leftrightarrow \alpha(H)$ is a nonzero integer for some root $\alpha \in R(M) \Leftrightarrow$ the orbit $K(p)$ is singular along $c$ in that $K(p)$ has a lower dimension than $K(c(t))$ for a generic point (a non-conjugate point) $c(t)$.

(ii) $c$ is closed $\Leftrightarrow$ there is a positive number $t_{o}$ such that $t_{o} \alpha(H)$ is an integer for every $\alpha \in R(M) \Leftrightarrow K_{(1)}$ fixes some point $c\left(t_{o}\right), t_{o}>0$.

(iii) If $K_{(1)}$ fixes the point $c(2)$ ( $p$ lies in a polar of $o$, for example), then the set $N^{\langle p\rangle}:=\{|\alpha(2 H)| \mid \alpha \in R(M), \alpha(H) \neq 0\}$ consists of integers.

(iv) If $K_{(1)}$ fixes $c(2)$ and if $c$ has no conjugate point of $o$ in the interval $] 0,1[$, then the set $N^{\langle p\rangle}$ is $\{1\},\{2\}$ or $\{1,2\}$.

(v) If $N^{\langle p\rangle}$ is one of $\{1\},\{2\}$ and $\{1,2\}$, then c has no conjugate point in $] 0,1[$ conversely. If furthermore $M$ is the bottom space $M \%$, then $p$ lies in a polar of $o$.

Proof is easy (See Section 2).

5.6a Remark. We mention a few words about 5.6. Assume $p$ lies in a polar $\mathrm{M}^{+}(p)$ of $o$ to consider the geometric implication of $N^{\langle p\rangle}$. Then the geodesic $c$ is entirely contained in the meridian $\mathrm{M}^{-}(p)$, since the symmetry $s_{o}$ reverses the tangent vector $c^{\prime}(1) \in T_{p} M$. $c$ meets a centriole $C(q)$ for $(1, p)$ in $M^{-}(p)$ (Cf. 4.6a) at the midpoint $q=c(1 / 2)$. Thus, if $N^{\langle p\rangle}=\{1\}, \operatorname{dim} M^{+}(p)$ is positive and $\operatorname{dim} C(q)=0$. If $N^{\langle p\rangle}=\{2\}$, one has $\operatorname{dim} M^{+}(p)=0$ (i.e., $p$ is a pole) and $\operatorname{dim} C(q)>0$. If $N^{\langle p\rangle}=\{1,2\}$, both $\operatorname{dim} M^{+}(p)$ and $\operatorname{dim} C(q)$ are positive.

5.6b Remark. We explain $N^{\langle p\rangle}$ in terms of the root system. If $N^{\langle p\rangle}=\{1\}$ (resp. $\{2\})$ in (iv), then $2 H$ (resp. $H$ ) equals $H^{j}$ for some simple root $\alpha_{j}$ such that the highest root $\alpha^{\sim}=\cdots+\alpha_{j}+\cdots$, where $H^{j}$ is the dual, $\left(2 /\left\|\alpha_{j}\right\|^{2}\right) \varpi_{j}$, of the $j$-th fundamental weight $\varpi_{j}$ and $K_{(1)}$ fixes $c(2)$ (resp. $p=c(1)$ ). If $N^{\langle p\rangle}=\{1,2\}$, then $2 H$ equals $H^{j}$ for some simple root $\alpha_{j}$ such that $\alpha^{\sim}=\cdots+2 \alpha_{j}+\cdots$ or else $H^{j}+H^{k}$ for $\alpha^{\sim}=\cdots+\alpha_{j}+\cdots+\alpha_{k}+\cdots$, $j<k$. Our main concern will be the last case of $N^{\langle p\rangle}=\{1,2\}$.

5.7 THEOREM. (i) Every simple Kähler space is either a polar or a centriole in a simple group. (ii) If a simple group $G$ has a pole, then some of the centrioles is Kählerian. 
More strongly, the Kählerian centrioles are exactly those which contain the midpoints $b$ of geodesic segments from 1 to the pole without having conjugate point of 1 at $b$.

Proof. (i) follows from (5.4.i), since a simple Kählerian space satisfies (5.4.iv). (ii) Let $c$ be an arbitrary geodesic in $G$ which passes through $1=c(0)$ and the pole $p=c(1)$ without having conjugate point at the midpoint $b=c(1 / 2)$. The orbit $(\operatorname{ad} G)(b)$ of the stabilizer $\operatorname{ad} G$ at 1 is regular; that is, the stabilizer $K$ of $b$ in $\operatorname{ad} G$ or its component $K_{(1)}$ is the centralizer of $H=c^{\prime}$. Since $K \subset \operatorname{ad}(G)$ does not exchange 1 and $p, K$ (does not reverse $H$ but) also fixes $H$. Therefore the centriole $(\operatorname{ad} G)(b)$ is Kählerian. Conversely, let $(\operatorname{ad} G)(b)=: G / K$ be a Kählerian centriole ( $\neq$ a singleton). Then the circle group $c$ in the center of $K \subset G$ passes through $b$ (by 5.8a below). The members of $\mathscr{L} K \subset \mathscr{L}$ ad(G) vanish on $c$. If $b$ is a conjugate point, then those members of $\mathscr{L} \operatorname{ad}(G)$ which vanish at $b$ make a subalgebra which contains $\mathscr{L} K$, contrary to the fact that $\mathscr{L} K$ is a maximal subalgebra in the simple $\mathscr{L} G$.

5.8 REMARK. (1) All the orbits $(\operatorname{ad} G)(c(t))$ of $\operatorname{ad} G, 0<t<1$, are Kählerian manifolds but not subspaces of $G$ except for $(\operatorname{ad} G)(b)$. (2) Some polar of a group $G$ in a local isomorphism class of simple groups is Kählerian if and only if $\alpha^{\sim}\left(H^{k}\right)=1$ for some $k$. See an example in 2.16. (3) Actually every simple Kählerian space is a centriole in a group as Table I (and/or Table II and 4.10 for $G_{2}\left(R^{n}\right)^{\sim}$ ) will indicate (See 5.12); EIII is a centriole in $T \cdot \mathrm{E}_{6}\left(\subset \mathrm{E}_{7}\right)$ which is not simple exceptionally.

5.8a REMARK. Let $b$ be a point of a space $M$ which a polar or a centriole $N$ contains. Take a shortest circle $c$ which contains $b$ and the origin. Then (5.6) through (5.6b) apply to $c$ with $H:=c^{\prime}$ and one can read off the possible root system with multiplicity of the $c$-orthogonal space $N^{\perp}$ to $N$ at $b$ by (2.15). If $M$ is a group, one has $N \fallingdotseq M / N^{\perp}$. If $N$ is Kählerian and $M$ is simple moreover, then $N^{\perp}$ contains a circle as the open subgroup of the center, which is $c$, since one sees $N^{\langle p\rangle}=\{1\}$ or $\{2\}$ in this case; See Section 2 for more details (noting that one may assume that $N$ is a polar by passing to the image of the projection in $4.4 \mathrm{~b}$ and 4.7 ). In particular $c$ passes through $b$ in the proof of (5.7).

The next theorem gives another reason why the polars of a group are evendimensional; the theorem says more and will be used in a substantial way.

5.9 THEOREM. Let $M$ be a subspace of $G$ through 1 . And let $M^{+}(p)$ be a polar of 1 in $M$. Then (i) there is a point $b$ in the meridian $M^{-}(p)$ such that $\beta:=\operatorname{ad}(b)$ fixes $p$, stabilizes the polar $G^{+}(p)$ in $G$ and defines a complex structure $J$ on the tangent space $T_{p} G^{+}(p)$; hence $\operatorname{dim} G^{+}(p)$ is even. (ii) If $M$ is given by a gr-involution $\tau$ of $G$ as $M=F\left(\tau \circ S_{1}, G\right)_{(1)}$, then the tangent space $T_{p} M^{+}(p)$ is totally real in $T_{p} G^{+}(p)$ with respect to $J$; in particular, one has $\operatorname{dim} M^{+}(p)=\frac{1}{2} \operatorname{dim} G^{+}(p)$. Moreover (iii) the automorphism $\beta$ of $G^{+}(p)$ carries $M^{+}(p)$ onto its c-orthogonal space in $G^{+}(p)$, fixing $p$.

Proof. Choose a point $b$ in $M^{-}(p)$ such that $b^{2}=p$; thus $b$ is pre-involutive. Since $\operatorname{ad}(p)$ equals $s_{1} \circ s_{p}, \operatorname{ad}(p)$ acts on $T_{p} G^{+}(p)$ as -1 , the negative identity as in the proof 
of (5.1). Thus $\beta$ acts on it as a complex structure $J ; \beta(p)=p$ and $J^{2}=\beta^{2}=-1$ on $T_{p} G^{+}(p)$. (ii) One has $\tau(b)=b^{-1}$, while $\tau$ fixes $p$ and so it does every point on the polar $M^{+}(p)$; $T_{p} M^{+}(p)=F\left(\tau, T_{p} G^{+}(p)\right)$. One has to show that $\beta$ carries $T_{p} M^{+}(p)$ onto its orthogonal complement in $T_{p} G^{+}(p)$, which is $F\left(-\tau, T_{p} G^{+}(p)\right)$ that is, the $(-1)$-eigenspace of $\tau$. Every tangent vector $X \in T_{p} M^{+}(p)$ satisfies $\tau \circ \beta(X)=\beta^{-1} \circ \tau(X)=\beta^{-1}(X)=-\beta(X)$; thus $X$ is carried into $F\left(-\tau, T_{p} G^{+}(p)\right)$ by $\beta$. (iii) $\beta$ stabilizes $G^{+}(p)$, since it does $T_{p} G^{+}(p)$. From its action on the tangent space $T_{p} G^{+}(p)$, one sees that $\beta$ carries $M^{+}(p)$ into its $c$-orthogonal space.

5.10 Corollary. Assume a subspace $M=G / K$ of a simple group $G$ has equal rank $r(M)=r(G)$ to $G$. If $G$ has a pole, then some of the centrioles in $M$ is an $R$-space.

Proof. $M$ contains a maximal torus of $G$, which implies that the pole $p$ of 1 in $G$ is also one in $M$. By (5.7), some centriole $C_{G}:=\operatorname{ad}(G)(b)$ is Kählerian, where $b$ is a midpoint of a minimal geodesic segment joining 1 to $p$ within $M$ which has the same root system $R(M)=R(G)$ by $r(M)=r(G)$ as $G$ (hence 5.7 applies). The corresponding centriole $C:=M \cap \operatorname{ad}(G)(b)$ is totally real in $\operatorname{ad}(G)(b)$ by (5.9) which one may apply by passing to a polar $\pi\left(C_{G}\right)$ with the double covering map $\pi$ which projects the two points 1 and $p$ into a singleton.

5.11 THEOREM. In the setting of Theorem 5.9, further assume that there is a circle $c$ in the meridian $\mathrm{M}^{-}(p)$ which contains $\{1, b, p\}$ and has no conjugate point of 1 at $b$ as a geodesic in $G$. Then

(i) the centriole $N:=C_{M}(b)$ through b for $(1, p)$ in $M^{-}(p)$ is an $R$-space;

(ii) the centriole $N^{c}:=C_{G}(b)$ through $b$ in $G^{-}(p)$ for $(1, p)$ is Kählerian;

(iii) $N^{c}$ is the complexification of $N$; and

(iv) the orbit ad $G(b)$ is a homogeneous Kählerian manifold.

Proof. $\quad C_{G}(b)$ is Kählerian by 5.7(ii), $b$ being the midpoint of a segment of $c$ between 1 and $p$ obviously. Now the double covering projection: $G^{-}(p) \rightarrow G^{-}(p) /\{1, p\}$ (See 4.4b) carries $C_{G}(b)$ and $C_{M}(b)$ onto polars in $G^{-}(p) /\{1, p\}$ and $M^{-}(p) /\{1, p\}$ respectively. And (5.7.ii) applies, giving the asserted (i) and (iii). (iv) The stabilizer of $b$ in $\operatorname{ad} G$ equals the one in $\operatorname{ad} G^{-}(p)$, and hence it is the centralizer of a circle group. Thus one obtains (iv) by H. C. Wang's theorem.

5.11a REMARK. The centriole $N$ in 5.11 has dimension $=0$ if $N^{\langle p\rangle}=\{1\}$ (See 5.6a). And so does $N^{c}$ then. If $N^{\langle p\rangle}=\{2\}$, then $M^{+}(p)$ and $G^{+}(p)$ are a pole of 1 in $M$ and $G$ respectively by $5.6 \mathrm{a}$.

5.12. In Table I, we list all the simple Kähler spaces $N^{C}$ on the second column and the $R$-spaces $N$ on the first column together with the natural inclusions: $N \rightarrow N^{C}$ as in 5.11; naturally we omit the Kähler spaces as $R$-spaces. The Kähler spaces are centrioles in the groups on the third column (Cf. 5.8). The arrows indicate monomorphisms and the diagrams are commutative. 
TABLE I. The $R$-spaces and the Kähler spaces.

\begin{tabular}{|c|c|c|c|c|c|}
\hline & $N$ & & $N^{c}$ & & $G$ \\
\hline 1 & $\begin{array}{c}\mathrm{SO}(n) \\
\uparrow\end{array}$ & $\rightarrow$ & $\underset{\uparrow}{\operatorname{DIII}(n)}$ & $\rightarrow$ & $\mathrm{SO}(2 n)$ \\
\hline 2 & $G_{p}\left(R^{n}\right)$ & $\rightarrow$ & $G_{p}\left(C^{n}\right)$ & $\rightarrow$ & $\mathrm{U}(n)$ \\
\hline 3 & $\begin{array}{l}\mathrm{UII}(n) \\
\uparrow\end{array}$ & $\rightarrow$ & $\underset{\uparrow}{\operatorname{DIII}(2 n)}$ & $\rightarrow$ & $\begin{array}{c}\mathrm{SO}(4 n) \\
\uparrow\end{array}$ \\
\hline 4 & $\begin{array}{c}\mathrm{U}(n) \\
\uparrow\end{array}$ & $\rightarrow$ & $\underset{\uparrow}{G_{n}\left(C^{2 n}\right)}$ & $\rightarrow$ & $\begin{array}{c}\mathrm{U}(2 n) \\
\uparrow\end{array}$ \\
\hline 5 & $\mathrm{UI}(n)$ & $\rightarrow$ & $\mathrm{CI}(n)$ & $\rightarrow$ & $\operatorname{Sp}(n)$ \\
\hline 6 & $\underset{\uparrow}{\operatorname{Sp}(n)}$ & $\rightarrow$ & $\underset{\uparrow}{\mathrm{CI}(2 n)}$ & $\rightarrow$ & $\underset{\uparrow}{\operatorname{Sp}(2 n)}$ \\
\hline 7 & $G_{p}\left(H^{n}\right)$ & $\rightarrow$ & $G_{2 p}\left(C^{2 n}\right)$ & $\rightarrow$ & $\operatorname{SU}(2 n)$ \\
\hline 8 & $S^{p} \cdot S^{n-p}$ & $\rightarrow$ & $\mathrm{G}_{2}\left(R^{n+2}\right)^{\sim}$ & $\rightarrow$ & $\mathrm{SO}(n+2)^{\sim}$ \\
\hline $1_{6}$ & $\begin{array}{c}\mathrm{SO}(6) \\
\downarrow\end{array}$ & $\rightarrow$ & $\begin{array}{c}\text { DIII(6) } \\
\downarrow\end{array}$ & $\rightarrow$ & $\begin{array}{c}\mathrm{SO}(12)^{\sim} \\
\downarrow\end{array}$ \\
\hline 9 & $\underset{\uparrow}{\operatorname{AII}(4)} / Z_{2}$ & $\rightarrow$ & $\begin{array}{c}\text { EVII } \\
\uparrow\end{array}$ & $\rightarrow$ & $\begin{array}{c}\mathbf{E}_{7} \\
\uparrow\end{array}$ \\
\hline 10 & $\underset{\uparrow}{G_{2}\left(H^{4}\right)^{\%}}$ & $\rightarrow$ & $\underset{\uparrow}{\text { EIII }}$ & $\rightarrow$ & $\begin{array}{c}T \cdot \mathrm{E}_{6} \\
\uparrow\end{array}$ \\
\hline $1_{5}$ & $\mathrm{SO}(5)$ & $\rightarrow$ & DIII(5) & $\rightarrow$ & $S O(10)^{\sim}$ \\
\hline $3_{3}$ & $\begin{array}{c}\mathrm{UII}(3) \\
\downarrow\end{array}$ & $\rightarrow$ & $\begin{array}{c}\operatorname{DIII}(6) \\
\downarrow\end{array}$ & $\rightarrow$ & $\begin{array}{c}\mathrm{SO}(12)^{\sim} \\
\downarrow\end{array}$ \\
\hline 11 & $\begin{array}{c}T \cdot \mathrm{EIV} \\
\uparrow\end{array}$ & $\rightarrow$ & $\begin{array}{c}\text { EVII } \\
\uparrow\end{array}$ & $\rightarrow$ & $\begin{array}{c}\mathrm{E}_{7} \\
\uparrow\end{array}$ \\
\hline 12 & $\begin{array}{c}\text { FII } \\
\uparrow\end{array}$ & $\rightarrow$ & $\underset{\uparrow}{\text { EIII }}$ & $\rightarrow$ & $\begin{array}{c}T \cdot \mathbf{E}_{6} \\
\uparrow\end{array}$ \\
\hline $8_{8,0}$ & $S^{8}$ & $\rightarrow$ & $G_{2}\left(R^{10}\right)^{\sim}$ & $\rightarrow$ & $\mathrm{SO}(10)^{\sim}$ \\
\hline
\end{tabular}

5.13 REMARK. From the table one may observe and can prove that every polar $N^{+}(p)$ in an $R$-space $N$ which is a subspace of the complexification $N^{C}$ is a (totally geodesic) $R$-subspace in the polar $N^{C+}(p)$ in $N^{C}$, a Kähler space.

5.14 REMARK on rotating. One can smoothly rotate the subspace $\mathrm{M}^{+}(p)$ within $G^{+}(p)$ in the setting of (5.9.iii) with a circle group $T$ generated by $\operatorname{ad}(H)$ (See 5.6 and/or 7.7) (if $G$ is simple); $T$ contains $\beta$. Similarly for the subspace $N$ of $N^{C}$ in 5.11 ; this fact about an $R$-space is known (2.23). The analogous fact was proven (Prop. 4.1 in [S]) 
for a $C$-space (or a totally complex subspace), by which we mean a local Kähler space $N$ in a quaternionic $\left(\boldsymbol{H}\right.$-)Kähler space $N^{H}$ such that an $\boldsymbol{H}$-scalar in $\mathrm{Sp}(1)$ in the isotropic subgroup at a point of $N$ rotates $N$ onto its $c$-orthogonal; for example, $G_{1}\left(C^{n}\right)$ in $G_{1}\left(H^{n}\right)$. For the other cases, geometric meanings are yet to know.

\section{Riemannian submersions.}

We will construct Riemannian submersions of a certain type out of polars by using results in the preceding section (and going out of the category of the symmetric spaces partly).

6.0 Assumptions. Let $M^{+}(p)$ be a polar of $o$ in $M=G / K ; M^{+}(p)=K_{(1)} / K^{+}$, (1.5a.ii). Let $c$ be a circle containing $\{o, p\}$ which has no conjugate point of $o$ on the open geodesic arc from $o$ to $p$, (5.6.iv). Let $C(q)$ denote the $K^{+}$-orbit through the midpoint $q=c(1 / 2)$ of the arc. We write $K / K^{+}$for $K_{(1)} / K^{+}$(without proving the equality) in this section.

Then $c$ lies on the meridian $M^{-}(p)=G^{-} / K^{+}$, because $s_{o} \circ s_{p}$ fixes $c$ pointwise. Since $p$ is a pole of $o$ in $M^{-}(p), C(q)$ is a centriole $K^{+} / K^{++}$for $(o, p)$ in $M^{-}(p)$. Thus we obtain a fibration

6.1 .

$$
C(q)=K^{+} / K^{++} \rightarrow K / K^{++} \rightarrow K / K^{+}=M^{+}(p)
$$

of the homogeneous manifold $K / K^{++}$(not a symmetric space) fibred by $C(q)$ over $M^{+}(p)$. We notice that all three, $C_{M}(q), K / K^{++}$and $M^{+}(p)$, are submanifolds of a single space $M . C_{M}(q)$ and $M^{+}(p)$ are subspaces of $M$.

The projection $f: K / K^{++} \rightarrow M^{+}(p)$ is a submersion, which we want to make Riemannian. The tangent space $T_{p} M^{+}(p)$ is spanned by the values $v(p)$ of the members $v$ of the Lie algebra $\mathfrak{f}=\mathscr{L} K$ which belong to some root $\alpha$ satisfying $|\alpha(H)|=1$, while $T_{q}\left(K / K^{++}\right)$is the direct sum of $T_{q} C(q)$, called the vertical space, and the subspace, called the horizontal space, spanned by the values $v(q)$ of the same members $v$ as above. $f$ carries the horizontal space at $q$ onto $T_{p} M^{+}(p)$ as a $K^{++}$-module isomorphism but not as an isometry if $K / K^{++}$is given the induced Riemannian metric from $M$. We can make it an isometry simply by doubling the induced metric on the horizontal space in view of the condition $|\alpha(H)|=1$. Thus one see that

6.2. the projection $f: K / K^{++} \rightarrow M^{+}(p)$ is a K-equivariant Riemannian submersion with respect to the modified metric; in particular $f$ is harmonic by a well-known theorem of Eells-Sampson, since the fibre is totally geodesic in $K / K^{++}$. It is also known (and will not be used either in this paper) that

6.2a. every "horizontal" harmonic (resp. minimal) map: $N \rightarrow K / K^{++}$composed with $f$ is also a harmonic (resp. minimal) map: $N \rightarrow M^{+}(p)$, (Eells-Wood). Here "horizontal" means that the image of the differential lies in the horizontal spaces. 
Under a stronger condition than non-conjugacy in (6.0), one easily obtains the following, the point of which is the fact that the projection $F$ is defined with the appropriate geodesics.

6.3 Proposition. If $\mathrm{K}^{++}$fixes $c$ (for example, if $\mathrm{K}^{++}$is connected, or if $c$ is minimal between $o$ and $p$ ) in (6.0), then

(i) the bundle $K / K^{++}$is a subbundle (of codimension one) of the bundle

$$
\left.C(q)=K^{+} / K^{++} \times\right] 0,2\left[\rightarrow B_{10,2[} \rightarrow K / K^{+}=M^{+}(p),\right.
$$

where $B_{I}$ denotes $\bigcup_{b \in K} b \circ c(I) \subset M$ for an interval $I$ in $[0,2[$ and the projection $F: B_{10,2[} \rightarrow M^{+}(p): b \circ c(t) \mapsto b \circ c(1)$ is defined by the geodesic arcs $b \circ c$;

(ii) the (well-defined) projection $F$ is $K$-equivariant; and

(iii) each $\left.B_{[t, t]}, t \in\right] 0,1[$, is a $K$-orbit through $c(t)$ and the restriction of (6.3a) to $B_{[t, t]}$ is a subbundle which is $K$-equivariantly isomorphic with $K / K^{++}=B_{[1 / 2,1 / 2]},(6.1)$; and

(iv) in case $M$ is the group $G$, the manifold $B_{[t, t]}$ is Kählerian and homogeneous for every $t \in] 0,1[$.

Proof. Easy and omitted. (iv) follows from (iii) and (5.11.iv).

6.3b COROLlaRY. Under the above assumptions (and in the notations),

(i) $B_{[1 / 2,1]}$ is a mapping cylinder of $f$ and $B_{[0,1]}$ is a mapping cone of $f$; thus $M^{+}(p)$ is a deformation retract of $B_{[1 / 2,1]}$; and hence

(ii) $K / K^{++}$and $C(q)$ are contractible to a point in $M$ and $\mathrm{M}^{-}(p)$ respectively.

We mention a simple application.

6.3c COROllary. No centriole in a Kählerian space is Kählerian.

6.4. In Table II, we will make a list of the fibrations (6.1) (or rather their local isomorphism classes), abbreviated to $K^{+} / K^{++} \rightarrow \rightarrow K / K^{+}$, which satisfy (6.0) and the condition $N^{\langle p\rangle}=\{1,2\}$ (that is, both $\operatorname{dim} K^{+} / K^{++}$and $\operatorname{dim} K / K^{+}$are positive. See $5.6 \mathrm{iv}$ ). We include $M$ and its root system with the multiplicities of the roots in Table II; for example, the first line indicates $G_{p}\left(C^{q}\right)=K^{+} / K^{++} \rightarrow \rightarrow G_{q}\left(C^{n}\right)=K / K^{+} \subset \mathrm{SU}(n)=M$ and the root system $R(M)=A_{n-1}, n-1=$ rank of $M$, with multiplicity $=2$, the numbers after the semicolon indicating the obvious specification. For a few more examples, $\left(\mathrm{C} / \mathrm{BC}_{r}, 2 m, 2,1 ; q\right)$ means $R(M)=\mathrm{C}_{r}$ or $\mathrm{BC}_{r}\left(R(M)=\mathrm{C}_{r}\right.$ iff $\left.m=0\right)$ and $2 m, 2$ and 1 are the multiplicities of the shortest roots, longer roots and the longest roots in this order. Those after semicolons in $\left(\mathrm{D}_{r}, 2 ; 1, r\right)$ and $\left(\mathrm{E}_{6}, 2 ; 3\right)$, etc. indicate that $2 H=H^{1}+H^{r}$ and $2 H=H^{3}$, etc.

6.4a REMARK. In Table II, one notes that there is a surjection of the set of the local classes of the fibrations $K^{+} / K^{++} \rightarrow \rightarrow K / K^{+}=M^{+}(p), M$ simple and not groups, onto the set of those with simple groups $M$; this is immediate from the existence of the Cartan monomorphism of $M=G / K$ into a covering group $G^{\wedge},(1.9)$. But the map is 
not injective for the same reason.

6.4b EXAMPLE. (1) Calabi's fibration DIII $(r) \rightarrow \mathrm{SO}(2 r+1) / \mathrm{U}(r) \rightarrow S^{2 r}$ appears in the local class $\left(\mathrm{B}_{r}, 2,2 ; r\right)$; he used it to prove that minimal immersions: $S^{2} \rightarrow S^{2 r}$ correspond to horizontal (6.2a) holomorphic maps: $S^{2} \rightarrow \mathrm{SO}(2 r+1) / \mathrm{U}(r)$. (2) $\left(\mathrm{BC}_{1}, 8,7\right)$ is one of the Hopf fibrations.

TABLE II. Fibrations over polars.

\begin{tabular}{|c|c|c|c|}
\hline $\begin{array}{l}\left(\mathrm{A}_{n-1}, 2 ; p, q\right) \\
\left(\mathrm{A}_{n-1}, 1 ; p, q\right) \\
\left(\mathrm{A}_{n-1}, 4 ; p, q\right) \\
\left(\mathrm{A}_{2}, 8 ; 1,2\right) \\
\left(\mathrm{B} / \mathrm{D}_{r}, 2,2 ; q\right)\end{array}$ & $\begin{array}{l}G_{p}\left(C^{q}\right) \\
G_{p}\left(R^{q}\right) \\
G_{p}\left(H^{q}\right) \\
S^{8} \\
\text { OIII }(q)\end{array}$ & $\begin{array}{l}\rightarrow \rightarrow G_{q}\left(C^{n}\right) \\
\rightarrow \rightarrow G_{q}\left(C^{n}\right) \\
\rightarrow \rightarrow G_{q}\left(H^{n}\right) \\
\rightarrow \rightarrow \text { FII } \\
\rightarrow \rightarrow G_{2 q}\left(R^{n}\right)\end{array}$ & $\begin{array}{ll}\subset \mathrm{SU}(n), & p<q<n . \\
\subset \mathrm{AI}(n), & p<q<n . \\
\subset \mathrm{AII}(n), & p<q<n . \\
\subset \mathrm{EIV} . & \\
\subset \mathrm{SO}(n), & q \leqq r, n=2 r+1 \text { or } 2 r .\end{array}$ \\
\hline$\left(\mathrm{B} / \mathrm{D}_{r}, m, 1 ; q\right)$ & $O(q)$ & $\rightarrow \rightarrow G_{q}\left(R^{m+r}\right) \times G_{q}\left(R^{r}\right)$ & $\subset G_{r}\left(R^{m+2 r}\right), \quad 0 \leqq m, q \leqq r$ \\
\hline $\begin{array}{l}\left(\mathrm{D}_{r}, 2 ; 1, r\right) \\
\left(\mathrm{D}_{r}, 1 ; 1, r\right)\end{array}$ & $\begin{array}{l}C P^{r-1} \\
R P^{r-1}\end{array}$ & $\begin{array}{l}\rightarrow \rightarrow \operatorname{DIII}(r) \\
\rightarrow \rightarrow \operatorname{SO}(r)\end{array}$ & $\begin{array}{l}\subset S O(2 r) \\
\subset G_{r}\left(R^{2 r}\right)\end{array}$ \\
\hline$\left(\mathrm{C}_{r}, 2,2 ; q\right)$ & $\mathrm{CI}(q)$ & $\rightarrow \rightarrow G_{q}\left(H^{r}\right)$ & $\subset \mathrm{Sp}(r), \quad 0<q<r$. \\
\hline$\left(\mathrm{C}_{r}, 1,1 ; q\right)$ & $\mathrm{UI}(q)$ & $\rightarrow \rightarrow G_{q}\left(C^{r}\right)$ & $\subset \mathrm{CI}(r), \quad 0<q<r$. \\
\hline$\left(\mathrm{C} / \mathrm{BC}_{r}, 2 m, 2,1 ; q\right)$ & $\mathrm{U}(q)$ & $\rightarrow \rightarrow G_{q}\left(C^{r}\right) \times G_{q}\left(C^{m+r}\right)$ & $\subset G_{r}\left(C^{m+2 r}\right), \quad 0 \leqq m, q \leqq r$. \\
\hline$\left(\mathrm{C} / \mathrm{BC}_{r}, 4 m, 4,3 ; q\right)$ & $\operatorname{Sp}(q)$ & $\rightarrow \rightarrow G_{q}\left(H^{r}\right) \times G_{q}\left(H^{m+\eta}\right)$ & $\subset G_{r}\left(H^{m+2 r}\right), \quad 0 \leqq m, q \leqq r$ \\
\hline$\left(\mathrm{C} / \mathrm{BC}_{r}, 4,4,1 ; q\right)$ & $\mathrm{UII}(q)$ & $\rightarrow \rightarrow G_{2 q}\left(C^{n}\right)$ & $\subset \operatorname{DIII}(n), \quad q \leqq r, n=2 r+1$ or $2 r$. \\
\hline $\begin{array}{l}\left(\mathrm{BC}_{2}, 8,6,1 ; 1\right) \\
\left(\mathrm{BC}_{2}, 8,6,1 ; 2\right)\end{array}$ & $\begin{array}{l}S^{1} \times S^{7} \\
S^{1}\end{array}$ & $\begin{array}{l}\rightarrow \rightarrow G_{2}\left(R^{10}\right)^{\sim} \\
\rightarrow \rightarrow \operatorname{DIII}(5)\end{array}$ & $\begin{array}{l}\subset \text { EIII. } \\
\subset \text { EIII. }\end{array}$ \\
\hline$\left(\mathrm{C}_{3}, 8,1 ; 2\right)$ & $S^{1} \times S^{9}$ & $\rightarrow \rightarrow$ EIII & $\subset$ EVII. \\
\hline$\left(C_{3}, 8,1 ; 1\right)$ & $S^{1}$ & $\rightarrow \rightarrow$ EIII & сEVII. \\
\hline$\left(\mathrm{BC}_{1}, 8,7\right)$ & $S^{7}$ & $\rightarrow \rightarrow S^{8}$ & $\subset$ FII. \\
\hline$\left(E_{6}, 2 ; 3\right)$ & $C P^{5}$ & $\rightarrow \rightarrow$ EII & $\subset \mathrm{E}_{6}$ \\
\hline$\left(E_{6}, 2 ; 2\right)$ & $S^{2}$ & $\rightarrow \rightarrow$ EII & $\subset \mathrm{E}_{6}$. \\
\hline$\left(\mathrm{E}_{6}, 2 ; 1,6\right)$ & $G_{2}\left(R^{10}\right)^{\sim}$ & $\rightarrow \rightarrow$ EIII & $\subset \mathbf{E}_{6}$. \\
\hline$\left(E_{6}, 1 ; 3\right)$ & $\boldsymbol{R} \boldsymbol{P}^{5}$ & $\rightarrow \rightarrow \mathrm{CI}(4)$ & $\subset$ EI. \\
\hline$\left(E_{6}, 1 ; 2\right)$ & $S^{1}$ & $\rightarrow \rightarrow \mathrm{CI}(4)$ & $\subset$ EI. \\
\hline$\left(E_{6}, 1 ; 1,6\right)$ & $S^{4} \times S^{4}$ & $\rightarrow \rightarrow G_{2}\left(H^{4}\right)^{\%}$ & $\subset \mathrm{EI}$. \\
\hline$\left(E_{7}, 2 ; 6\right)$ & $G_{2}\left(R^{12}\right)^{\sim}$ & $\rightarrow \rightarrow$ EVI & $\subset \mathrm{E}_{7}$. \\
\hline$\left(E_{7}, 2 ; 1\right)$ & $S^{2}$ & $\rightarrow \rightarrow$ EVI & $\subset \mathrm{E}_{7}$. \\
\hline$\left(E_{7}, 2 ; 2\right)$ & $C P^{7}$ & $\rightarrow \rightarrow \mathrm{EV} \%$ & $\subset \mathbf{E} \%$. \\
\hline$\left(E_{7}, 1 ; 6\right)$ & $S^{5} \times S^{5}$ & $\rightarrow \rightarrow G_{4}\left(C^{8}\right)^{\%}$ & $\subset \mathrm{EV}$. \\
\hline$\left(E_{7}, 1 ; 1\right)$ & $S^{1}$ & $\rightarrow \rightarrow G_{4}\left(C^{8}\right)^{\%}$ & $\subset \mathbf{E V}$ \\
\hline$\left(E_{7}, 1 ; 2\right)$ & $\boldsymbol{R} \boldsymbol{P}^{7}$ & $\rightarrow \rightarrow \mathrm{AI}(8) / Z_{4}$ & $\subset \mathrm{EV} \%$. \\
\hline$\left(E_{8}, 2 ; 8\right)$ & $S^{2}$ & $\rightarrow \rightarrow$ EIX & $\subset \mathrm{E}_{8}$ \\
\hline$\left(E_{8}, 2 ; 1\right)$ & $G_{2}\left(R^{16}\right)^{\sim}$ & $\rightarrow \rightarrow$ EVIII & $\subset \mathrm{E}_{8}$. \\
\hline$\left(E_{8}, 1 ; 8\right)$ & $S^{1}$ & $\rightarrow \rightarrow \operatorname{DIII}(8)^{\%}$ & $\subset$ EVIII. \\
\hline$\left(E_{8}, 1 ; 1\right)$ & $S^{7} \times S^{7}$ & $\rightarrow \rightarrow G_{8}\left(R^{16}\right) \#$ & $\subset$ EVIII. \\
\hline$\left(F_{4}, 2,2 ; 1\right)$ & $S^{2}$ & $\rightarrow \rightarrow$ FI & $\subset \mathbf{F}_{4}$ \\
\hline$\left(F_{4}, 2,2 ; 4\right)$ & $G_{2}\left(R^{9}\right)^{\sim}$ & $\rightarrow \rightarrow$ FII & $\subset F_{4}$. \\
\hline$\left(F_{4}, 1,1 ; 1\right)$ & $S^{1}$ & $\rightarrow \rightarrow S^{2} \cdot \mathrm{CI}(3)$ & $\subset$ FI. \\
\hline$\left(F_{4}, 1,1 ; 4\right)$ & $S^{3} \times S^{4}$ & $\rightarrow \rightarrow G_{1}\left(H^{3}\right)$ & $\subset$ FI. \\
\hline
\end{tabular}


TABLE II. (Continued).

\begin{tabular}{llll}
\hline$\left(\mathrm{F}_{4}, 2,1 ; 1\right)$ & $S^{1}$ & $\rightarrow \rightarrow S^{2} \cdot G_{3}\left(C^{6}\right)$ & $\subset$ EII. \\
$\left(\mathrm{F}_{4}, 2,1 ; 4\right)$ & $S^{3} \times S^{5}$ & $\rightarrow \rightarrow G_{2}\left(C^{6}\right)$ & $\subset$ EII. \\
$\left(\mathrm{F}_{4}, 4,1 ; 1\right)$ & $S^{1}$ & $\rightarrow \rightarrow S^{2} \cdot \mathrm{DIII}(6)$ & $\subset \mathrm{EVI}$. \\
$\left(\mathrm{F}_{4}, 4,1 ; 4\right)$ & $S^{3} \times S^{7}$ & $\rightarrow \rightarrow G_{4}\left(R^{12}\right)^{\sim}$ & $\subset \mathrm{EVI}$. \\
$\left(\mathrm{F}_{4}, 8,1 ; 1\right)$ & $S^{1}$ & $\rightarrow \rightarrow S^{2} \cdot \mathrm{EVII}$ & $\subset \mathrm{EIX}$. \\
$\left(\mathrm{F}_{4}, 8,1 ; 4\right)$ & $S^{3} \times S^{11}$ & $\rightarrow \mathrm{EVI}$ & $\subset \mathrm{EIX}$. \\
$\left(\mathrm{G}_{2}, 2,2\right)$ & $S^{2}$ & $\rightarrow \rightarrow \mathrm{GI}$ & $\subset \mathrm{G}$. \\
$\left(\mathrm{G}_{2}, 1,1\right)$ & $S^{1}$ & $\rightarrow \rightarrow S^{2} \cdot S^{2}$ & $\subset \mathrm{GI}$. \\
\hline
\end{tabular}

\section{Graded Lie algebras.}

In this section we will show that the simple graded Lie algebras

$$
\text { 7.1. } \quad \mathfrak{g}^{\prime}=\mathfrak{g}_{-2}^{\prime}+\mathfrak{g}_{-1}^{\prime}+\mathfrak{g}_{0}^{\prime}+\mathfrak{g}_{1}^{\prime}+\mathfrak{g}_{2}^{\prime}, \quad\left[\mathfrak{g}_{1}^{\prime}, \mathfrak{g}_{1}^{\prime}\right]=\mathfrak{g}_{2}^{\prime} \neq 0
$$

as studied by Kaneyuki ( $[\mathrm{K}],[\mathrm{KA}])$ correspond bijectively to the triplets $(\mathfrak{g}, \tau, H)$ of a compact simple Lie algebra $\mathfrak{g}$, its involution $\tau$ and a member $H$ of $\mathfrak{g}$ satisfying $\tau(H)=-H \neq 0,(7.6)$, and also to the pairs of the local classes of fibrations discussed in Section 6 and satisfying certain conditions, (7.10); we will exclude the cases of complex Lie algebras $\mathfrak{g}^{\prime}$. The condition $\left[\mathfrak{g}_{1}^{\prime}, \mathfrak{g}_{1}^{\prime}\right]=\mathfrak{g}_{2}^{\prime} \neq 0$ in (7.1) is equivalent to $\mathfrak{g}_{1}^{\prime} \neq 0 \neq \mathfrak{g}_{2}^{\prime}$ and to $\mathfrak{g}_{-1}^{\prime} \neq 0 \neq \mathfrak{g}_{-2}^{\prime}$.

We begin with recalling necessary facts.

7.2. The graded algebra $\mathfrak{g}^{\prime}$ contains a unique member $Z$ such that each linear subspace $\mathfrak{g}_{p}^{\prime}$ is characterized as the eigenspace of $\operatorname{ad}(Z)$ for the eigenvalue $p,[\mathrm{~K}]$.

Hence $\mathfrak{g}^{\prime}$ is not compact, and we will turn to the compact form $\mathfrak{g}$.

7.3. $g$ admits an involution $\tau$ such that $\mathfrak{f}:=F(\tau, \mathfrak{g})$ is isomorphic with a "maximal compact" subalgebra of $\mathfrak{g}^{\prime}$.

Thus if $\mathfrak{g}=\mathfrak{f}+\mathfrak{m}$ denotes the symmetry decomposition for $\tau$ where $\mathfrak{m}$ denotes $F(-\tau, \mathfrak{g})$, then one could write $\mathfrak{g}^{\prime}=\mathfrak{f}+i \mathfrak{m}, i^{2}=-1$.

7.4. Since $i m$ contains $Z$, there is a unique member $H$ of $g$ defined by $Z=i H$. One has $\tau(H)=-H$.

Using $H$, we obtain these decompositions:

7.5 $\quad g=\mathfrak{g}_{\mathrm{ev}}+\mathfrak{g}_{[1]} \quad$ with $\mathfrak{g}_{\mathrm{ev}}:=\mathfrak{g}_{[0]}+\mathfrak{g}_{[2]}$,

where $\mathfrak{g}_{[p]}$ is the eigenspace of $\operatorname{ad}(H)^{2}$ for the eigenvalue $-p^{2}$. These are symmetry decompositions $(1.6 \& 1.6 \mathrm{a})$ of $g$ and $g_{\mathrm{ev}}$ respectively, which are non-trivial in that none of $\mathfrak{g}_{[1]}, \mathfrak{g}_{[0]}$ and $\mathfrak{g}_{[2]}$ vanishes.

We also obtain these non-trivial symmetry decompositions by 7.4 : 
7.5 $\quad \mathfrak{i}=\mathfrak{f}_{\mathrm{ev}}+\mathfrak{f}_{[1]} \quad$ with $\mathfrak{f}_{\mathrm{ev}}=\mathfrak{f}_{[0]}+\mathfrak{f}_{[2]}$,

where $\mathfrak{f}_{[p]}$ also equals $\mathfrak{t} \cap \mathfrak{g}_{[p]}$.

The converse obtains also; we record this easy fact below.

7.6 Proposition. The real simple graded Lie algebras 7.1 correspond bijectively with the triple systems $(\mathrm{g}, \tau, H)$ satisfying the following conditions, modulo obvious equivalence: (1) $g$ is a compact simple Lie algebra with a non-trivial involution $\tau$, (2) the member $H$ of $\mathrm{g}$ satisfies $\tau(H)=-H$ and (3) the eigenvalues of $\operatorname{ad}(H)^{2}$ are $0,-1^{2}$ and $-2^{2}$.

In fact, the graded Lie algebra (7.1) is recovered as $\mathfrak{g}^{\prime}=F(\tau, \mathfrak{g})+i F(-\tau, \mathfrak{g})$ and the eigenspace $\mathfrak{g}_{p}^{\prime}$ for the eigenvalue $p$ of $\operatorname{ad}(i H)$ acting on $\mathfrak{g}^{\prime}$. The equality $\left[\mathfrak{g}_{1}^{\prime}, \mathfrak{g}_{1}^{\prime}\right]=\mathfrak{g}_{2}^{\prime}$ is an immediate consequence of $[\mathfrak{m}, \mathfrak{m}]=\mathfrak{f}$ for a symmetry decomposition $\mathfrak{g}=\mathfrak{f}+\mathfrak{m}$ of a simple Lie algebra $\mathfrak{g}$. That $\mathfrak{g}_{2}^{\prime}$ does not vanish follows from (3); $\operatorname{since} \operatorname{ad}(H)$ is skew-symmetric (w.r.t. the Killing form), the eigenvalue $p$ of $\operatorname{ad}(i H)$ has equal multiplicity to $-p$.

Now we want to give a geometric interpretation to $(g, \tau, H)$ which satisfies the conditions in (7.6).

7.7. Thus let $G$ denote a connected group with Lie algebra $g$ on which $\tau$ can act; $G=G \%$, for instance. We denote $F(\tau, G)$ by $K$ and its c-orthogonal space at 1 by $M$; i.e., $M=F\left(\tau \circ s_{1}, G\right)_{(1)} \fallingdotseq G / K$ (This $\fallingdotseq$ can be replaced with $=$ if $\left.G=G \%\right) . H$ is tangent to $M$ at 1 by $\tau(H)=-H$. We set up the root system (5.5) of $M$ and see that the set $N^{\langle p\rangle}$ in (5.6 iv) is $\{1,2\}$. Hence the situation fits $(6.0) ; p:=c(1)$ lies in a polar $M^{+}(p) \subset G^{+}(p)$ at least if $G=G \%$ (and hence $M=M^{\%}$ ), where $c(t):=\exp (t \pi H)(1)$. Note that we still have $N^{\langle p\rangle}=\{1,2\}$ if we use a root system of $G$.

Hence we have two fibrations of type (6.1) by the non-conjugacy in (5.6.v). We will indicate the correspondence between the subgroups in (6.1) and those in the present setting by these diagrams:

$$
\begin{array}{ll}
7.8_{\mathrm{G}} & G_{\mathrm{ev}} / G_{[0]} \rightarrow G / G_{[0]} \rightarrow G / G_{\mathrm{ev}}=G^{+}(p) \text { and } \\
7.8_{M} & K_{\mathrm{ev}} / K_{[0]} \rightarrow K / K_{[0]} \rightarrow K / K_{\mathrm{ev}}=M^{+}(p) .
\end{array}
$$

In fact, the tangent space $T_{p} M^{+}(p)$ is isomorphic with $\mathfrak{f}_{[1]}$ as a $\mathfrak{f}_{\text {ev }}$-module (by the local isomorphism $M \fallingdotseq G / K)$ and so is $T_{p} G^{+}(p)$ with $\mathfrak{g}_{[1]}$ as a $g_{\mathrm{ev}}-\operatorname{module} M^{+}(p)$ may be written as $K / K_{\mathrm{ev}}$ and $G^{+}(p)$ as $G / G_{\mathrm{ev}}$, where $K_{\mathrm{ev}}$ and $G_{\mathrm{ev}}$ are the stabilizers with Lie algebras $\mathfrak{f}_{\mathrm{ev}}$ and $\mathrm{g}_{\mathrm{ev}}$ respectively. The centrioles $C_{G}(q)$ and $C_{M}(q)$ in the meridians $G^{-}(p)$ and $M^{-}(p)$ can be written as above, $G_{[0]}$ denoting a subgroup with Lie algebra $g_{[0]}$ and so forth.

7.9 LEMMA. In the setting (7.7) with (7.6), (i) the centriole $C_{G}(q)$ is Kählerian; (ii) Theorem 5.9 applies to the pair of the polars $G^{+}(p)$ and $M^{+}(p)$; (iii) Theorem 5.11 applies to the pair of the centrioles $C_{G}(q)$ and $C_{M}(q)$; and (iv) Proposition 6.3 applies to the 
fibrations $7.8_{G}$ and $7.8_{M}$.

Proof. One has (i) by Theorem 5.7 and the non-conjugacy in (5.6.v). (5.9) clearly applies in the present setting. (5.11) applies by the non-conjugacy again. (6.3) applies by (i) and (5.7).

In particular, Theorem 5.9 gives

7.9a. Every point $b$ of $G / G_{[0]}$ defines a complex (isometric) structure $J_{b}$ on the tangent space $T_{\operatorname{pr}(b)} G^{+}(p)$ to the base space $G^{+}(p)$ of the fibration pr: $G / G_{[0]} \rightarrow G^{+}(p)$, 7.8, at the point $\operatorname{pr}(b)=b^{2}$ by the restriction $J_{b}$ of $\operatorname{ad}(b)$ to $T_{\operatorname{pr}(b)} G^{+}(p)$. And the map: $b \mapsto J_{b}$ is $\operatorname{ad}(G)$-equivariant.

We have established the first half (i) of the next theorem:

7.10 THEOREM. (i) Given a simṕle graded Lie algebra 7.1, or equivalently a triple system $(\mathfrak{g}, \tau, H)$ subject to the conditions in 7.6, there corresponds a double tiered fibration:

$$
\begin{array}{cc}
7.10_{G} & C_{G}(q) \rightarrow \operatorname{ad}(G)(q) \rightarrow G^{+}(p) \\
\uparrow & \uparrow \uparrow \\
7.10_{M} & C_{M}(q) \rightarrow \operatorname{ad}(K)(q) \rightarrow M^{+}(p)
\end{array}
$$

within a compact connected simple group $G$ with $\mathscr{L} G=\mathfrak{g}$ and within the space $M=F\left(\tau \circ s_{1}, G\right)_{(1)}$, the component through 1 , defined with a gr-involution $\tau$ of $G$ respectively, where

(a) $G^{+}(p)$ and $M^{+}(p)$ are polars of 1 in $G$ and $M$ respectively;

(b) $C_{G}(q) \ni q$ is a Kählerian centriole for $(1, p)$ in $G^{-}(p)$ and is the complexification of the centriole $C_{M}(q)$ for $(1, p)$ in $M^{-}(p), \operatorname{dim} C_{M}(q)=\operatorname{dimg}_{2}^{\prime}>0,\left(C_{M}(q)\right.$ is an $R$-space);

(c) every point b of $\operatorname{ad}(G)(q)$ defines a complex structure on the tangent space to $G^{+}(p)$ at the projection $b^{2}$ of $b$ and $\operatorname{ad}(b)$ rotates $M^{+}(p)$ onto its c-orthogonal space at $b^{2}$ fixing $b^{2}$ (and stabilizing $\left.G^{+}(p)\right), \operatorname{dim} M^{+}(p)\left(=\operatorname{dim} g_{1}^{\prime}\right)>0$;

(d) the vertical arrows indicate the inclusions induced by that of $M$ into $G$; and

(e) the bundle manifold $\operatorname{ad}(G)(q)$ can be deformed to $\operatorname{ad}(G)$-equivariantly isomorphic bundle $\operatorname{ad}(G)(c(t))$ as in (6.3) (especially, the points move along the pre-determined geodesics at constant speed in the deformation), and similarly for $\operatorname{ad}(K)(q)$.

(ii) Conversely, every two-tiered fibration $\left(7.10_{G}, 10_{M}\right)$ satisfying (a) through (e) comes up, as above, from a simple graded algebra (7.1) or a triple system $(\mathrm{g}, \tau, H)$ in 7.6.

Proof. It remains to prove (ii). By (b) and (5.7), one can recover the circle $c$ with $c^{\prime}=H$. $c$ has no conjugate point of 1 between 1 and $p$ except at $p$ by (e). Therefore $N^{\langle p\rangle}$ in (5.6.iv) is contained in $\{1,2\}$. The dimension conditions in (b) and (c) give the equality $N^{\langle p\rangle}=\{1,2\}$. Finally $\tau$ satisfies $\tau(H)=-H$, since the circle group $c$ in $M$ passes through 1.

7.10a Remark. The condition (e) cannot be dropped to prove the converse in 
(7.10), as these two examples show:

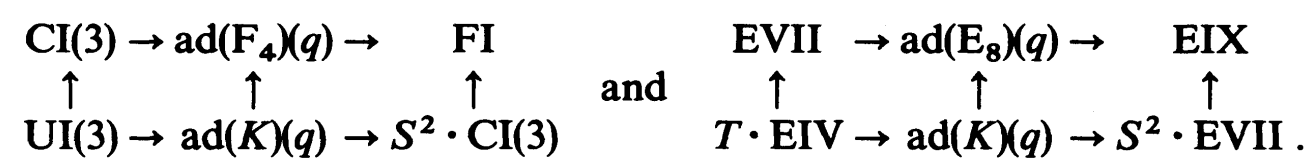

For these, $N^{\langle p\rangle}$ contains 3.

7.11. We want to understand some facts of geometry of the gradation 7.1. The case $\mathfrak{g}_{-2}^{\prime}=0=\mathfrak{g}_{2}^{\prime}$ is already known [KN]. In that case, $\mathfrak{g}^{\prime}$ is the Lie algebra of the transformation group $G^{\prime}$ of an $R$-space $M$ such that $G^{\prime}$ is the subgroup of the holomorphic transformation group of the complexification $M^{c}$ of $M$ which leaves $M$ invariant, having $\mathfrak{g}_{0}^{\prime}+\mathfrak{g}_{1}^{\prime}$ as that of the isotropy subgroup at a point $o$. $g_{1}^{\prime}$ acts trivially on the tangent space $T_{o} M$. For instance, if $M$ is a sphere [resp. a real projective space], then $G^{\prime}$ is the conformal [resp. the projective] transformation group. Since $G^{\prime}$ is also characterized as a connected simple group which acts on a compact symmetric space $M$ and contains $I(M)_{(1)}\left(\neq G^{\prime}\right)$ properly (See [N24]), one cannot expect a similar interpretation of Kaneyuki's graded algebra 7.1, but we make the next observation.

7.12 Proposition. Let $G^{\prime}$ be a Lie group with finite center whose Lie algebra is given the gradation 7.1. Then (i) one has a fibration

$$
G_{\mathrm{ev}}^{\prime} / G_{0}^{\prime} \rightarrow G^{\prime} / G_{0}^{\prime} \rightarrow G^{\prime} / G_{\mathrm{ev}}^{\prime}
$$

with symmetric spaces as the fibre and the base, where the connected subgroups $G_{\mathrm{ev}}^{\prime}$ and $G_{0}^{\prime}$ have the Lie algebras $\mathfrak{g}_{-2}^{\prime}+\mathfrak{g}_{0}^{\prime}+\mathfrak{g}_{2}^{\prime}$ and $\mathrm{g}_{0}^{\prime}$ respectively; (ii) the invariant pseudo-Riemannian metric of the space $G^{\prime} / G_{\mathrm{ev}}^{\prime}$ has signature equal to zero; (iii) the subspace $G_{1,2}^{\prime} / G_{2}^{\prime}$ has the tangent space contained in the light cone (i.e., the tangent vectors are isotropic) in the tangent space to $G^{\prime} / G_{\mathrm{ev}}^{\prime}$ (similarly for $\left.G_{-1,-2}^{\prime} / G_{-2}^{\prime}\right), G_{1,2}^{\prime}$ and $G_{2}^{\prime}$ having the Lie algebras $\mathfrak{g}_{1}^{\prime}+\mathfrak{g}_{2}^{\prime}$ and $\mathfrak{g}_{2}^{\prime}$ respectively; (iv) similarly to (ii) and (iii) for $G_{\mathrm{ev}}^{\prime} / G_{0}^{\prime}$ and its subspace $G_{0,2}^{\prime} / G_{0}^{\prime}$; (v) the subspace $G_{1,2}^{\prime} / G_{2}^{\prime}$ is flat; and (vi) $G^{\prime} / G_{\mathrm{ev}}^{\prime}$ is $K$-diffeomorphic with the normal bundle of its subspace $K / K_{\mathrm{ev}}$, where $K$ denotes a maximal compact subgroup of $G^{\prime}$.

Proof. (i) Obvious. (ii) One has $\operatorname{dim} G^{\prime} / G_{\mathrm{ev}}^{\prime}=2 \operatorname{dim} K / K_{\mathrm{ev}}$ by 7.9 and the tangent space to the subspace $K / K_{\mathrm{ev}}$ is a maximal one on which the metric defined by the Killing form $B$ of $\mathfrak{g}^{\prime}$ is negative definite. Hence the signature (=index) equals zero. (iii) The tangent space to $G_{1,2}^{\prime} / G_{2}^{\prime}$ may be identified with $g_{1}^{\prime}$ on which $B=0$ by 7.2 and the invariance property of $B$. (The space $G_{1,2}^{\prime} / G_{2}^{\prime}$ is not semisimple because $G_{1,2}^{\prime}$ is a Heisenberg group.) Similar arguments give (iv). (v) follows from $\left[\mathfrak{g}_{2}^{\prime}, \mathfrak{g}_{2}^{\prime}\right]=0=\left[\mathfrak{g}_{2}^{\prime}, \mathfrak{g}_{1}^{\prime}\right]$ for the graded algebra $g^{\prime}$; the vector fields in $g_{2}^{\prime}$ must vanish identically on $G_{1,2}^{\prime} / G_{2}^{\prime}$. (vi) the orthogonal complement $T_{o}^{\perp} K / K_{\mathrm{ev}}$ to $T_{o} K / K_{\mathrm{ev}}$ in $T_{o} G^{\prime} / G_{\mathrm{ev}}^{\prime}$ is tangent to the noncompact form of $K / K_{\mathrm{ev}}$ on which the exponential map is a diffeomorphism.

7.12a Remark. One could make 7.12 more complete by proving the converse; 
for that one may need more necessary conditions. The pairs of subspaces in the light cones (iii $\&$ iv) are dual to each other with $B$ as the canonical pairing; they are isotropy-invariant subspaces in (iv) but not in (iii). The next proposition will imply that the manifolds in the fibration (7.12.i) are all contained in $G^{\prime}$ (in fact they are $\operatorname{ad}\left(G^{\prime}\right)$-orbits); thus it will become more relevant to the structure of $G^{\prime}$ itself.

7.13 Proposition. In the notations of 7.7, further assume that the rank $r(K)$ equals $r(G)$ and that $K=F(\tau, G)$ is connected. Then (i) the fibration $\left(7.8_{M}\right)$ is realized within $K$; and (ii) $G^{\prime}$ is isomorphic with the normal bundle $T^{\perp} K$ to $K$ in $G$ as a $K \times K$-manifold, where $G^{\prime}$ is the non-compact form that shares $K$ with $G$. And (iii) $G^{\prime} / G_{\mathrm{ev}}^{\prime}$ and $G_{\mathrm{ev}}^{\prime} / G_{0}^{\prime}$ are locally isomorphic with subspaces of $G^{\prime}$ which are the normal bundles of their subspaces $K / K_{\mathrm{ev}}$ and $K_{\mathrm{ev}} / K_{[0]}$ respectively.

Proof. (i) The point $p$ of $M \subset G$ is involutive and satisfies $\tau(p)=p^{-1}$. Hence $p=\tau(p)$ lies in $K$ by the assumption; one has $K^{+}(p)=M^{+}(p)$ by (1.5a.ii) or (4.4a). The geodesic $c$ lies in the meridian $M^{-}(p) \subset G^{-}(p)$. By the assumption on the rank and by (4.6b), $G^{-}(p)$ has equal rank to $K^{-}(p)$. Hence the tangent vector $H$ is carried into $T_{1} K$ by a member $\gamma$ of the isotropy subgroup for $G^{-}(p)$ at 1 . Thus $\gamma$ carries $c$ into a geodesic $c_{K}$ in $K^{-}(p)$. Since $\gamma$ fixes the pole $p$ in $G^{-}(p)$ by $(4.6 \mathrm{a})$, one sees that $c_{K}$ joins 1 to $p$. Therefore the manifolds in the fibration $\left(7.8_{M}\right)$ lie in $K$, since $c_{K}$ is congruent with $c$. (ii) The normal space $T_{1}^{\perp} K$ at 1 can be identified with $i m$ which is isomorphic with $T_{1}^{\perp} K$ as a $K$-module. Since the exponential map restricted to $i m$ is a $K$-equivariant diffeomorphism onto $M^{\prime} \subset G^{\prime}$, one can further identify $T_{1}^{\perp} K$ with $M^{\prime}$. So far one has a $K$-diffeomorphism of $T_{1}^{\perp} K \cup K \subset T^{\perp} K$ into $G^{\prime}$. One easily sees that this extends uniquely to a $K \times K$-equivariant diffeomorphism $\phi: T^{\perp} K \rightarrow G^{\prime}$. (iii) $G^{\prime} / G_{\mathrm{ev}}^{\prime}\left[\mathrm{resp} . G_{\mathrm{ev}}^{\prime} / G_{0}^{\prime}\right]$ is locally isomorphic with the $\operatorname{ad}(G)$-orbit through $p$ [resp. $\left.c_{K}(1 / 2)\right]$ which is a subspace of $G^{\prime}$. See (7.12.vi) for the rest.

7.14. We close this section by classifying the graded algebras (7.1) in our formulation $\left(7.8_{G}\right)$ plus $\left(7.8_{M}\right)$ specified with the symbols in Table II, thereby reconfirming Kaneyuki's classification in [K]. He labels them with (c1) through (e23) along with the complex ones (e24) through (e34) which we omit; we just indicate the correspondence between his and ours below and in Table III. For instance, the graded algebra (c1) corresponds $\left(\mathrm{A}_{n-1}, 2 ; p, q\right)+\left(\mathrm{A}_{n-1}, 1 ; p, q\right)$, which means (c1) corresponds to the fibration:

$$
G_{p}\left(C^{q}\right) \rightarrow G / G_{[0]} \rightarrow G_{q}\left(C^{n}\right)
$$

in $G=\mathrm{SU}(n)$ paired with the fibration:

$$
G_{p}\left(R^{q}\right) \rightarrow K / K_{[0]} \rightarrow G_{q}\left(R^{n}\right)
$$

in $\mathrm{AI}(n)=\mathrm{SU}(n) / \mathrm{SO}(n)$ by Theorem 7.10; the second one is obtained as the fixed point sets of $\tau$ acting on the space above each, which is the complex conjugation and gives 


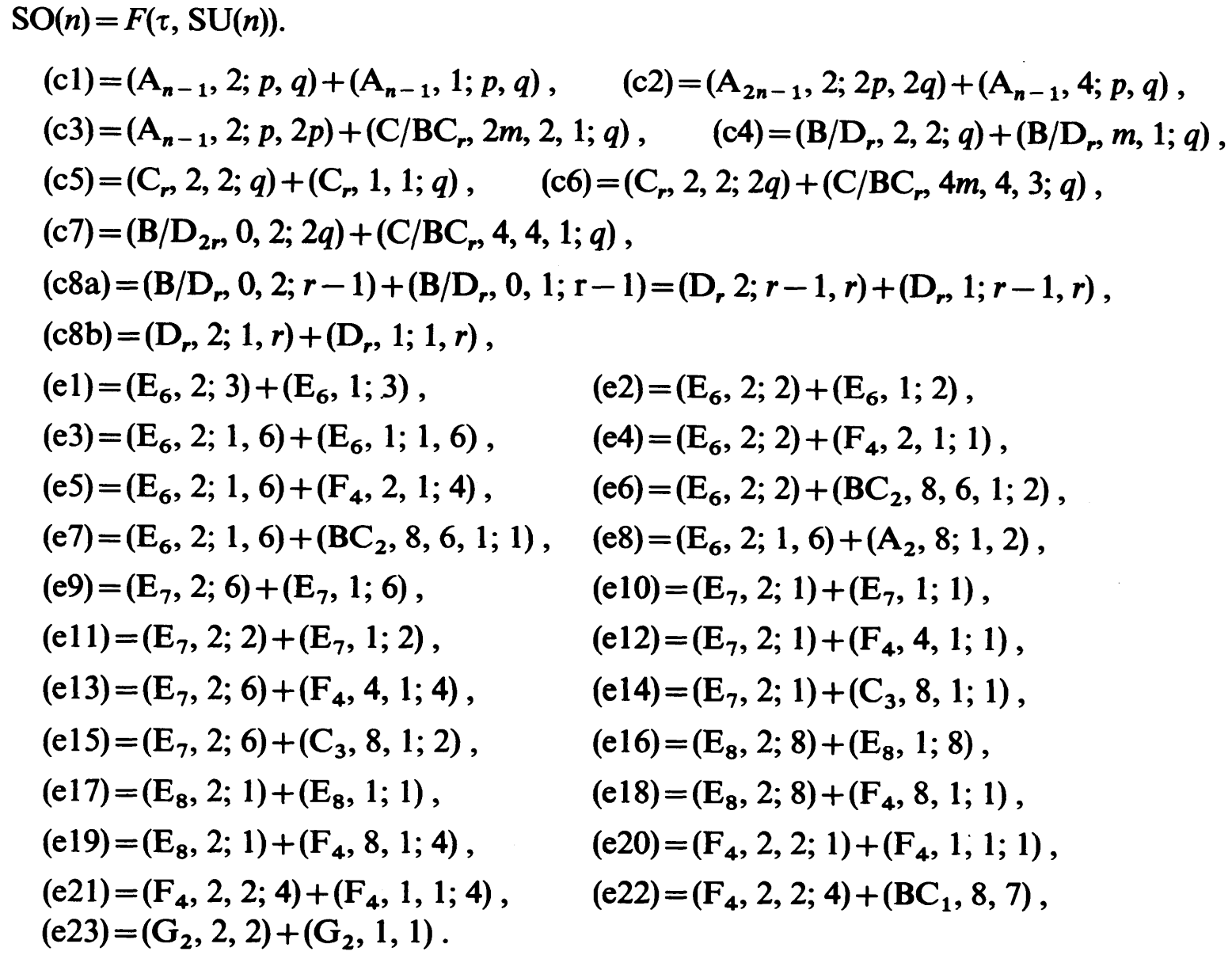

In Table III, we arrange those fibrations $\left(7.8_{G}\right)$ and $\left(7.8_{M}\right)$ on a line for each graded algebra whose label appears at the end of the line. The bundle manifolds are omitted as in Table II.

TABLE III. Fibrations $\left(7.8_{G} \& 8_{M}\right)$ for the graded Lie algebras.

\begin{tabular}{|c|c|c|c|c|}
\hline$G_{s}\left(C^{r}\right)$ & $\rightarrow \rightarrow G_{r}\left(C^{n}\right)$ & $G_{s}\left(R^{r}\right)$ & $\rightarrow \rightarrow G_{r}\left(R^{n}\right)$ & (c1) \\
\hline$G_{2 s}\left(C^{2 r}\right)$ & $\rightarrow \rightarrow G_{2 r}\left(C^{2 n}\right)$ & $G_{s}\left(H^{r}\right)$ & $\rightarrow \rightarrow G_{r}\left(H^{n}\right)$ & (c2) \\
\hline$G_{r}\left(C^{2 r}\right)$ & $\rightarrow \rightarrow G_{2 r}\left(C^{n}\right)$ & $\mathrm{U}(\boldsymbol{r})$ & $\rightarrow \rightarrow G_{r}\left(C^{p}\right) \times G_{r}\left(C^{q}\right), \quad p+q=n$ & (c3) \\
\hline $\operatorname{DIII}(r)$ & $\rightarrow \rightarrow G_{2 r}\left(R^{n}\right)$ & $\mathrm{SO}(r)$ & $\rightarrow \rightarrow G_{r}\left(R^{q}\right) \times G_{r}\left(R^{q}\right), \quad p+q=n$ & (c4) \\
\hline $\mathrm{CI}(r)$ & $\rightarrow \rightarrow G_{r}\left(H^{n}\right)$ & $\mathrm{UI}(r)$ & $\rightarrow \rightarrow G_{r}\left(C^{n}\right)$ & (c5) \\
\hline $\mathrm{CI}(2 r)$ & $\rightarrow \rightarrow G_{2 r}\left(H^{n}\right)$ & $\operatorname{Sp}(r)$ & $\rightarrow \rightarrow G_{r}\left(H^{p}\right) \times G_{r}\left(H^{q}\right)$ & (c6) \\
\hline $\operatorname{DIII}(2 r)$ & $\rightarrow \rightarrow G_{4 r}\left(R^{2 n}\right)$ & $\mathrm{UII}(r)$ & $\rightarrow \rightarrow G_{2 r}\left(C^{n}\right)$ & (c7) \\
\hline $\operatorname{DIII}(n-1)$ & $\rightarrow \rightarrow G_{2}\left(R^{2 n}\right)^{\sim}$ & $\mathrm{SO}(n-1)$ & $\rightarrow \rightarrow S^{n-1} \times S^{n-1}$ & (c8a) \\
\hline$C P^{n-1}$ & $\rightarrow \rightarrow \operatorname{DIII}(n)$ & $R P^{n-1}$ & $\rightarrow \rightarrow \mathrm{SO}(n)$ & (c8b) \\
\hline$C P^{5}$ & $\rightarrow \rightarrow$ EII & $R P^{5}$ & $\rightarrow \rightarrow \mathrm{CI}(4)$ & (e1) \\
\hline$S^{2}$ & $\rightarrow \rightarrow$ EII & $S^{1}$ & $\rightarrow \rightarrow \mathrm{CI}(4)$ & $(\mathrm{e} 2)$ \\
\hline
\end{tabular}


TABLE III. (Continued).

\begin{tabular}{|c|c|c|c|c|}
\hline$G_{2}\left(R^{10}\right)^{\sim}$ & $\rightarrow \rightarrow$ EIII & $S^{4} \times S^{4}$ & $\rightarrow \rightarrow G_{2}\left(H^{4}\right)^{\%}$ & (e3) \\
\hline$S^{2}$ & $\rightarrow \rightarrow \mathrm{EII}$ & $S^{1}$ & $\rightarrow \rightarrow S^{2} \cdot G_{3}\left(C^{6}\right)$ & (e4) \\
\hline$G_{2}\left(R^{10}\right)^{\sim}$ & $\rightarrow \rightarrow$ EIII & $S^{3} \times S^{5}$ & $\rightarrow \rightarrow G_{2}\left(C^{6}\right)$ & (e5) \\
\hline$S^{2}$ & $\rightarrow$ EII & $S^{1}$ & $\rightarrow \rightarrow \operatorname{DIII}(5)$ & (e6) \\
\hline$G_{2}\left(R^{10}\right)^{\sim}$ & $\rightarrow \rightarrow \mathrm{EIII}$ & $S^{1} \times S^{7}$ & $\rightarrow \rightarrow G_{2}\left(R^{10}\right)^{\sim}$ & (e7) \\
\hline$G_{2}\left(R^{10}\right)^{\sim}$ & $\rightarrow \rightarrow$ EIII & $S^{8}$ & $\rightarrow \rightarrow$ FII & (e8) \\
\hline$G_{2}\left(R^{12}\right)^{\sim}$ & $\rightarrow \rightarrow \mathrm{EVI}$ & $S^{5} \times S^{5}$ & $\rightarrow \rightarrow G_{4}\left(C^{8}\right)$ & (e9) \\
\hline$S^{2}$ & $\rightarrow \rightarrow \mathrm{EVI}$ & $S^{1}$ & $\rightarrow \rightarrow G_{4}\left(C^{8}\right)$ & (e10) \\
\hline$C P^{7}$ & $\rightarrow \rightarrow \mathrm{EV}$ & $S^{7}$ & $\rightarrow \rightarrow \mathrm{AI}(8)$ & (e11) \\
\hline$S^{2}$ & $\rightarrow \rightarrow \mathrm{EVI}$ & $S^{1}$ & $\rightarrow \rightarrow S^{2} \cdot \operatorname{DIII}(6)$ & (e12) \\
\hline$G_{2}\left(R^{12}\right)^{\sim}$ & $\rightarrow \rightarrow$ EVI & $S^{3} \times S^{7}$ & $\rightarrow \rightarrow G_{4}\left(R^{12}\right)^{\sim}$ & (e13) \\
\hline$S^{2}$ & $\rightarrow \rightarrow$ EVI & $S^{1}$ & $\rightarrow \rightarrow$ EIII & (e14) \\
\hline$G_{2}\left(R^{12}\right)^{\sim}$ & $\rightarrow \rightarrow \mathrm{EVI}$ & $S^{1} \times S^{9}$ & $\rightarrow \rightarrow$ EIII & (e15) \\
\hline$S^{2}$ & $\rightarrow \rightarrow$ EIX & $S^{1}$ & $\rightarrow \rightarrow \operatorname{DIII}(8)^{\%}$ & (e16) \\
\hline$G_{2}\left(R^{16}\right)^{\sim}$ & $\rightarrow \rightarrow$ EVIII & $S^{7} \times S^{7}$ & $\rightarrow \rightarrow G_{8}\left(R^{16}\right)^{\#}$ & (e17) \\
\hline$S^{2}$ & $\rightarrow \rightarrow$ EIX & $S^{1}$ & $\rightarrow \rightarrow S^{2} \cdot \mathrm{EVII}$ & (e18) \\
\hline$G_{2}\left(R^{16}\right)^{\sim}$ & $\rightarrow \rightarrow$ EVIII & $S^{3} \times S^{11}$ & $\rightarrow \rightarrow$ EVI & (e19) \\
\hline$S^{2}$ & $\rightarrow \rightarrow \mathrm{FI}$ & $S^{1}$ & $\rightarrow \rightarrow S^{2} \cdot \mathrm{CI}(3)$ & (e20) \\
\hline$G_{2}\left(R^{9}\right)^{\sim}$ & $\rightarrow \rightarrow$ FII & $S^{3} \times S^{4}$ & $\rightarrow \rightarrow G_{1}\left(H^{3}\right)$ & (e21) \\
\hline$G_{2}\left(R^{9}\right)^{\sim}$ & $\rightarrow \rightarrow$ FII & $S^{7}$ & $\rightarrow \rightarrow S^{8}$ & (e22) \\
\hline$S^{2}$ & $\rightarrow \rightarrow$ GI & $S^{1}$ & $\rightarrow \rightarrow S^{2} \cdot S^{2}$ & (e23) \\
\hline
\end{tabular}

\section{The twistor structures on symmetric spaces.}

R. Bryant $[\mathrm{Br}]$ proved that the twistor space $Z$ (in his sense) over a compact 1-connected symmetric space $N$ is characterized as the fibration 7.8 with $Z=G / G_{[0]}$ and $N=G^{+}(p) ; G / G_{[0]}$ is indeed a twistor space by (7.9a). He (and S. Salamon independently) classified all those twistor spaces; he picked up the fibrations in Table II which are contained in the simple groups. He also found that the complexification of $g$ has the gradation 7.1.

\section{References}

[ABS] M. Atryah, R. Bott and A. Shapiro, Clifford modules, Topology 3 Suppl. 1 (1964), 3-38.

[AHS] M. AtiYAH, N. Hitchin and I. Singer, Self-duality in four dimensional Riemannian manifolds, Proc. Roy. Soc. London A 362 (1978), 425-461.

[B] N. BouRBaKI, Groupes et Algèbres de Lie, IV, $V \& V I$, Hermann (1968).

[Br] R. L. BRYANT, Lie groups and twistor spaces, Duke Math. J. 52 (1985), 223-261.

[CS] J. B. CARrell and A. J. Sommese, Some topological aspects of $C^{*}$ actions on compact Kähler 
manifolds, Comm. Math. Helv. 54 (1979), 567-582.

[H] S. Helgason, Differential Geometry, Lie Groups and Symmetric Spaces, Academic Press (1978).

[K] S. KaneYukI, On the subalgebras $g_{0}$ and $g_{e v}$ of semisimple graded Lie algebras, J. Math. Soc. Japan 45 (1993), 1-19.

[KA] S. KANEYuKI and H. Asano, Graded Lie algebras and generalized Jordan triple systems, Nagoya Math. J. 112 (1988), 81-115.

[KN] S. Kobayashi and T. Nagano, On filtered Lie algebras and geometric structures, II, J. Math. Mech. 14 (1965), 513-522.

[MT] K. MASHIMO and H. TASAKI, Stability of closed Lie subgroups in compact Lie groups, Kodai Math. J. 13 (1990), 181-203.

[N24] T. NaGano, Transformation groups on compact symmetric spaces, Trans. Amer. Math. Soc. 118 (1965), 428-453.

[N67] T. Nagano, The involutions of compact symmetric spaces, II, Tokyo J. Math. 15 (1992), 39-82.

[NS] T. NAGANO and M. SUMI, The spheres in symmetric spaces, Hokkaido Math. J. 20 (1991), 331-352.

[S] M. SUMI, Stability of minimal submanifolds in symmetric spaces, to appear in Tsukuba J. Math.

[Tk] M. TAKEUCH, Cell decompositions and Morse equalities on certain symmetric spaces, Proc. Fac. Sci. Univ. Tokyo 12 (1965), 81-192.

[Ts] H. TASAKI, Quaternionic submanifolds in quaternionic symmetric spaces, Tôhoku Math. J. 38 (1986), 513-538.

[T] I-H. Tsal, Rigidity of proper holomorphic maps between symmetric domains, J. Differential Geom. 37 (1993), 123-160.

Added in proof. We should have explained the relevance of these papers below. [C] J. H. Cheng, Graded Lie algebras of the second kind. Trans. AMS 302 (1987), 467-488.

[T86] M. Takeuchi, Totally complex submanifolds of quaternionic symmetric spaces. Japan. J. Math. 12 (1986), 161-189.

[G] S. Gomyo, The construction of exceptional simple graded Lie algebras of the second kind. Proc. Japan Acad. 71 (1955), 13-16.

Present Addresses:

TADashi Nagano

DePartment of Mathematics, Sophia Univeesity,

CHIYODA-KU, TOKYO, 102 JAPAN.

Makiko Sumi Tanaka

Division of Natural Sciences, College of Liberal Arts, International Christian University, Mitaka, TOKYo, 181 JAPAN. 